\title{
Biphasic constitutive laws for biological interface evolution
}

\author{
P. Büchler, D. P. Pioletti, L. R. Rakotomanana
}

Abstract A model of tissue differentiation at the bone-implant interface is proposed. The basic hypothesis of the model is that the mechanical environment determines the tissue differentiation. The stimulus chosen is related to the bone-implant micromotions. Equations governing the evolution of the interfacial tissue are proposed and combined with a finite element code to determine the evolution of the fibrous tissue around prostheses. The model is applied to the case of an idealized hip prosthesis.

\section{Introduction}

The long-term stability of implants is a major concern in joint arthroplasties. Formation of a fibrous interface between the bone and the implant is a frequent problem. The fibrous interface looses the implant, which commonly causes pain and eventually leads to the implant failure.

The biological factors causing the growth of the fibrous interface are not completely clarified. Some studies pointed out the role of wear particles on bone osteolysis (Goodman 1994; Pioletti et al. 1999). Aspenberg and Herbertsson (1996) found that the mechanical environment of the bone cells is more important for the fibrous formation process than the presence of particles. Mechanical variables are assumed to be involved in the formation of the fibrous tissue like the hydrostatic compression (Skripitz and Aspenberg 2000; van der Vis et al. 1999), the fluid pressure (van der Vis et al. 1998a, b), and the fluid velocity (Prendergast et al. 1997; Yuan et al. 2000). In the present study, we assume that the most important parameter is the bone-implant micromotions. We know from the traditional twostages procedure used for the endosseous dental implant that the tissue found at the bone-implant interface is related to the loading history of the implant, There is a direct bone apposition on the implant for an unloaded implant, whereas the interfacial tissue is fibrous for implant loaded immediately after surgery (Brunski et al. 1979; Levy et al. 1996). The importance of micromotions on fibrous tissue formation is highlighted in the study of Farron et al. (1995). Using a finite element model of the knee, a strong correlation was found between the micromotions around a tibia plateau calculated with and the mean thickness of the radiolucent lyses observed around prostheses implanted into patients. The in vivo study of Jasty et al. (1997) showed that small amplitudes of micromotion $(\leq 20 \mu \mathrm{m})$ have no influence on the bone healing, but amplitudes greater than $150 \mu \mathrm{m}$ produce a fibrous interface at a depth of 1 or $2 \mathrm{~mm}$ around the implant during the 6 weeks following the implantation. Søballe et al. (1992a, b) confirmed these results and showed the reversibility of the process.

The biological processes considered in the present study include a first phase corresponding to the bone necrosis following the surgical procedure and a second phase of healing. The bone necrosis occurs at a depth of a few millimeters around the implant but the bone structure is

Received: 28 May 2002 / Accepted: 10 November 2002

P. Büchler ( $₫)$, D. P. Pioletti

LRO, Bat. AAB, Laboratory of Orthopedic Research,

EPFL, 1015 Lausanne, Switzerland

e-mail: philippe.buechler@epfl.ch

Tel.: +41-21-6938351; Fax: +41-21-6938660

L. R. Rakotomanana

IRMAR, Campus de Beaulieu, University of Rennes 1, 35042 Rennes, France

P. Büchler, D. P. Pioletti

Orthopaedic Hospital, Avenue Pierre-Decker 4,

1005 Lausanne, Switzerland 
not significantly modified. During the healing of the bone trauma, mesenchymal stem cells migrate in the region of the trauma and proliferate. We assume that the differentiation of these cells depends on mechanical factors. For low micromotions, the cells differentiate into osteoblasts (cells making bone) while for high micromotions, cells differentiate into fibroblasts (cells making fibrous tissue). The goal of the present study is to propose a stimulus and a law of evolution to describe the differentiation process (bone or fibrous tissue formation) at the bone-implant interface.

The second goal of this study is to propose a numerical implementation of the fibrous tissue formation law. Some numerical models of the bone density remodeling exist (Terrier et al. 1997; Weinans et al. 1994), but only Weinans et al. (1990) have proposed a two-dimensional numerical model of the fibrous tissue evolution around implants. The numerical model proposed in this study is used to evaluate the fibrous tissue formation around an idealized hip implant.

\section{Constitutive laws}

\section{Definition of the system}

The system considered in this study is considers the tissue around the implant. During the biological evolution, mass can be added or removed from the system. This notion of open system was first introduced in biomechanics by Cowin and Hegedus (1976). In this study, the tissue was considered as a bioactive composite made of bone and fibrous tissue undergoing finite deformations. Following the continuum assumption, the two constituents forming the composite are allowed to occupy the same position of physical space. In a given volume, each constituent participate to the bulk density. A specific volume $V_{T}$ of the composite is the sum of the volume of the fibrous tissue $V_{f}$ and of the bone $V_{b}$. We define a new internal variable $\kappa=V_{f} / V_{T}$ as the volume occupied by the fibrous tissue divided by the total volume. With this definition, the density $\rho$ of the body is given by:

$\rho=\kappa \rho_{f}+(1-\kappa) \rho_{b}$

where $\rho_{f}$ and $\rho_{b}$ are the densities of the fibrous tissue and of the bone, respectively. This definition implies that for $\kappa=0$ the tissue is bone and for $\kappa=1$ the tissue is fibrous. Cells present into the tissue actively modified the composition of the composite. Depending on mechanical stimuli, cells will produce either fibrous tissue or mineralized bone. This process modifies the volume fraction $\kappa$ of the composite. The evolution of $\kappa$ expresses then the tissue differentiation. Mass changes are induced by this differentiation process. Mass changes are due to the presence of a blood perfusant that brings to the cells the nutriment and the basic constituents needed to produce the matrix as well as exports the waste cells.

With the internal variable $\kappa$ defined above, the strain-energy functions are given by:

$$
\begin{aligned}
& W=\kappa W_{f}+(1-\kappa) W_{b} \\
& \rho \omega=\kappa \rho_{f} \omega_{f}+(1-\kappa) \rho_{b} \omega_{b}
\end{aligned}
$$

where $W$ is the strain energy per unit volume, and $\omega$ per unit mass. Equations (2) and (3) are simple formulations of strain-energy functions for a composite of two solids. Some assumptions on the nature of the composite have been made. Equations (2) and (3) are obviously valid for $\kappa=0$ and $\kappa=1$. For $0<\kappa<1$, the composite is considered as an homogenous solid having mechanical properties proportional to the volume fraction of each of its constituents. No coupling effect between the strain-energy functions of the two components is considered in this formulation.

A necessary and sufficient condition for existence of a solution is the quasiconvexity of the strain energy. Unfortunately, quasiconvexity is very difficult to interpret and verify, mainly because it is a global requirement over the entire domain of the solid (Ball 1977). For this reason, a more pragmatic way for verifying constitutive models may be adopted. It consists of checking monotonicity of laws in some particular situations: simple traction or simple elongation, isotropic dilatation, and simple shear as proposed by Curnier (1994).

\section{Law of evolution}

The formulation of the stimulus driving the evolution is of central interest for the remodeling model. Experimental studies have shown that the fibrous tissue formation is related to the micromotions at 
the bone-implant interface (Brunski et al. 1979; Farron et al. 1995; Jasty et al. 1997; Levy et al. 1996; Søballe et al. 1992a, b). The mechanical contact between the bone and the implant is complex but it can be decomposed into two major types of contacts. The first situation is a pure sliding contact. In this case the micromotion is defined as the displacement of the implant relative to the bone matrix. The second situation is a perfectly bounded contact. In this case the micromotions correspond to the deformation of the bone matrix at a certain depth around the implant (Fig. 1). The two different types of contact may probably occur simultaneously in different regions of the interface in clinical situation. The hypothesis chosen in this study is that the deformation of the bone matrix is the stimulus of the tissue differentiation. Since the principal mode of deformation is shear, the shear strain in the tissue is chosen as tissue differentiation stimulus. This type of stimulus was already proposed by Carter et al. $(1988,1998)$ and used to study tissue differentiation (Giori et al. 1995).

The general mathematical formulation of the stimulus is based on the decomposition of the right Cauchy-Green tensor $C$ into a purely volumetric contribution and a purely distortional contribution. According to ideas which go back to Flory (Flory, 1961), we may perform a multiplicative decomposition of $C$ into a dilatational part $C_{v o l}$ and a volume preserving part $C_{i s o}\left(\operatorname{det} C_{i s o} \equiv 1\right)$. Thus,

$C=C_{v o l} C_{i s o}$,

where

$\boldsymbol{C}_{\text {vol }}=J^{2 / 3} \boldsymbol{I}$

$C_{\text {iso }}=J^{-2 / 3} C$,

Where $I$ denotes the second-order identity tensor.

With this definition, we perform the deviatoric projection, proposed by Holzapfel, and calculate the deviatoric stress in the finite strain domain (Holzapfel 2000). A general form of stimulus in the finite strain theory is, for example, a scalar function of the first and second invariants of $C_{i s o}$ according to

$\varepsilon=\hat{\varepsilon}\left(\operatorname{tr} C_{i s o}, \operatorname{tr} C_{i s o}^{2}\right)$

This function $\varepsilon$ corresponds to the shear strain in the tissue and represents the tissue differentiation stimulus. With this definition of the remodeling stimulus, the law defining the evolution of the tissue is given by the following differential equation:

$\frac{d \kappa}{d t}=-v(\kappa-M(\varepsilon))$

where $v$ determines the rate of the biological evolution and $M(\varepsilon)$ is the driving function defined in Fig. 2. This function is introduced to account for the thresholds of the biological process. For shear strain lower than $\varepsilon_{\min }$ the driving function value is 0 (evolution in the direction of bone formation) and for shear strain greater than $\varepsilon_{\max }$ the function value is 1 (evolution in the direction of a fibrous tissue). $\varepsilon_{\min }$ and $\varepsilon_{\max }$ are the octahedric strains in the material corresponding to the experimentally observed micromotions of $20 \mu \mathrm{m}$ and $150 \mu \mathrm{m}$ respectively.
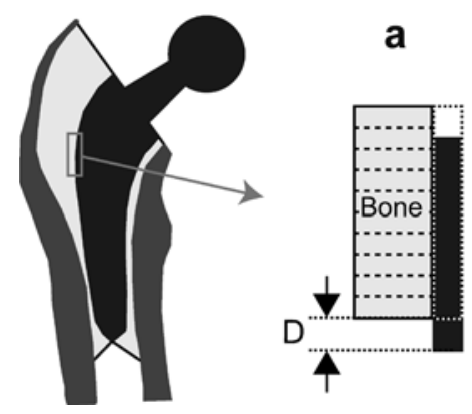

b

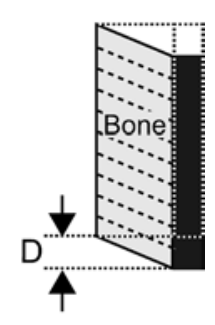

Fig. 1a, b. Details of the bone/implant interface. a represents a situation of pure sliding at the interface; $\mathbf{b}$ represents the same global micromotion $\mathrm{D}$ with an interface perfectly bonded. In the case b, the bone matrix and the cell in the bone matrix are deformed. This deformation is the stimulus considered in this study 


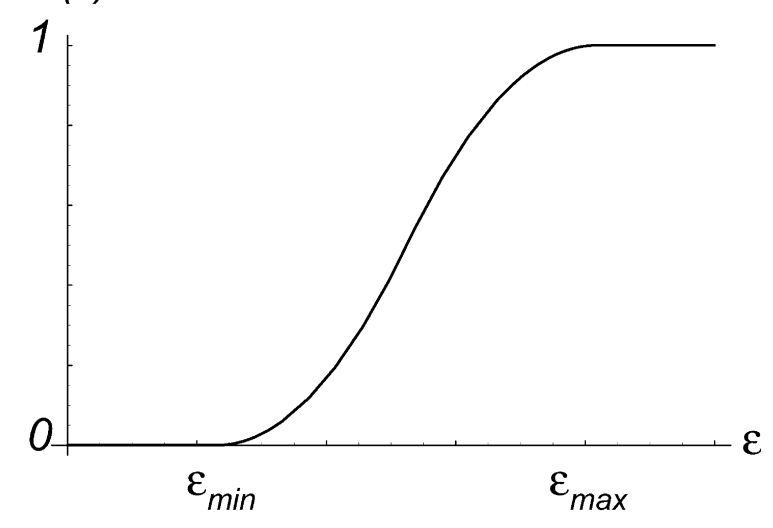

Fig. 2. Definition of the driving function $M(\varepsilon)$

\section{Conservation laws}

\section{Balance of mass}

For classical solids, the mass $m$ is conserved during the deformation process. This is not true in the present case, the mass variation is due to the adaptive process. The rate of change of the mass $m$ in the volume $\varphi(\Omega)$ in the deformed configuration is given by:

$\frac{d m}{d t}=\frac{d}{d t} \int_{\varphi(\Omega)} \rho d \omega=\int_{\varphi(\Omega)}\left(\frac{d \rho}{d t}+\rho \operatorname{div} \mathbf{v}\right) d \omega$

where the mapping $\varphi: \mathbf{y}=\varphi(\mathbf{x})$ describes the deformation, $\mathbf{v}$ is the spatial velocity field and $\operatorname{div}(\bullet)$ denotes the spatial divergence of $(\bullet)$. The rate of change of the mass is equal to the rate of mass supply $\dot{\boldsymbol{\Gamma}}$ due to the biological process, i.e.

$\frac{d \rho}{d t}+\rho \operatorname{div} \mathbf{v}=\dot{\Gamma}$

\section{Conservation of linear momentum}

The classical balance equation for momentum must be modified to include these mass transfers. The balance of the linear momentum of the volume $\varphi(\Omega)$ is given by:

$\rho \frac{d \mathbf{v}}{d t}+\dot{\Gamma} \overrightarrow{\mathbf{v}}=\operatorname{div} \mathbf{T}+\rho \mathbf{b}$

where $T$ is the Cauchy stress tensor and $\mathbf{b}$ is the body force per unit mass.

The left hand side of (10) represents the rate of change of the linear momentum $\rho \mathbf{v}$, while the right hand side corresponds to the sum of the external forces. A new internal force $\dot{\Gamma} \mathbf{v}$ appears. This force expresses the change of linear momentum caused by the mass apposition during the evolution.

\section{Conservation of angular momentum}

The rate of change of the angular momentum is balanced by the momentum of the external forces. In case of mass transfer, this relation results in the symmetry of the stress tensors

$\boldsymbol{T}=\boldsymbol{T}^{T}$ and $S=S^{T}$

where $S$ is the second Piola-Kirchhoff tensor defined as $T=J^{-1} F S F^{T}$ with $F$ the deformation gradient, and $J=\operatorname{det} F$.

\section{Energy conservation}

The total rate of change of the energy (internal and external) balances the total supply of energy through external forces (contact and volume) and the energy supply through the mass supply 
$\rho \frac{d e}{d t}=S: \dot{\boldsymbol{E}}-\dot{\Gamma}\left(e+\frac{\overrightarrow{\mathbf{v}}^{2}}{2}\right)$

where $E$ is the Green-Lagrange strain tensor. Within a thermodynamic regime incorporating thermal variables such as $\theta$ and $\eta$, the introduced strain-energy function (eq. (3)) is commonly referred as the Helmhotz free-energy function. Thus, a Legendre transformation gives (Holzapfel, 2000):

The first term at the right hand side of (12) is the stress power, while the second term corresponds to the energy transfer due to mass apposition or removal from the system during the biological evolution. If the mass of the system is constant, the equation reduces to its classical form.

\section{Thermodynamic restrictions}

The second law of thermodynamics states that the entropy production is always positive or equal to zero:

$\theta \rho \frac{d \eta}{d t}+\theta \eta \dot{\Gamma} \geq 0$

where $\eta$ is the specific entropy of the composite and $\theta$ its temperature.

$\omega=e-\theta \eta$

and, by using (12) the entropy inequality becomes:

$-\rho \frac{d \omega}{d t}-\dot{\Gamma}\left(\omega+\frac{\mathbf{v}^{2}}{2}\right)+\boldsymbol{S}: \dot{\boldsymbol{E}} \geq 0$

The second term in (15) represents the part of the energy transfer due to the mass change that does not contribute to the entropy production.

Quasi-static approximation

Next we discuss the quasi-static approximation. This assumption for deformation that occurs corresponds to a $\mathbf{v} \rightarrow 0$, which simplifies the equations of conservation. The balance of mass (Eq. 9) reduces then to:

$\frac{d \rho}{d t}=\dot{\Gamma}=\left(\rho_{f}-\rho_{b}\right) \frac{d \kappa}{d t}$

where the last equality is obtained by introducing the definition of the density $\rho$ (Eq. 1). The balance of linear momentum (Eq. 10), and the conservation of energy (Eq.12) become:

$\operatorname{div} T=0$

$\rho \frac{d e}{d t}=S: \dot{\boldsymbol{E}}-e\left(\rho_{f}-\rho_{b}\right) \frac{d \kappa}{d t}$

where, without loss of generality, also the body force $\mathbf{b}$ was omitted in Eq. (17). The last term on the right hand side of (18) corresponds to the internal energy produced by mass changes associated with the biological activity.

With the quasi-static approximation, the second law of thermodynamic becomes:

$\gamma=\gamma_{m e c}+\gamma_{b i o l} \geq 0$

$\gamma_{m e c}=\left(S-\rho \frac{\partial \phi}{\partial \boldsymbol{E}}\right): \dot{\boldsymbol{E}}$

$\gamma_{b i o l}=\left(\left(\rho_{b}-\rho_{f}\right) \omega-\rho \frac{\partial \omega}{\partial \kappa}\right) \frac{d \kappa}{d t}$

where $\gamma_{m e c}$ is the expression for the entropy production during the mechanical deformation of a solid and $\gamma_{b i o l}$ represents the entropy production due to the biological process. 
For, an isothermal elastic process we have then:

$\boldsymbol{S}=\rho \frac{\partial \omega}{\partial \boldsymbol{E}}$

$\gamma_{b i o l} \geq 0$

Expression (22) corresponds to the classical expression for the stress tensor derived from the strainenergy function. The relation (23) induces an apparent paradox. Biological processes tend towards order and structure inducing a negative entropy production. However, biological systems are open, so the production of negative entropy is localized on a limited region of the system. The global entropy production due to the biological activity is then positive. This is due to the interaction of the biological part of the system with the rest of the universe. The relation (23) expresses the direction of the biological evolution.

\section{Applications to orthopedic situations}

\section{Numerical algorithm}

Simulation of orthopedic situations have to incorporate complex geometries, such as bone or implants, as well as complex loading conditions. Numerical methods are the only means for handling this kind of problems. The present adaptation model is based on two types of equations. First the mechanical equilibrium Eqs. (17) and (11) that define a boundary value problem, and second the bone evolution Eq. (7) that describes an initial-value problem. Since the characteristic time of the adaptation process is much higher than the characteristic time of the deformation, the equations of equilibrium are the same as for classical materials. Usual methods for solving these equations may be used. In this study we used the commercial package ABAQUS Standard/5.8-1. The resolution of the initial-value problem is based on a modified Runge-Kutta method with an adaptation step size control proposed by Cash and Karp (Press et al. 1993). A schematic description of the numerical procedure used is presented in Fig. 3 We confirmed the results given in Press et al. (1993) that this method is roughly a factor of two more efficient than algorithms based on step doubling.

\section{Mathematical expression of the stimulus}

The exact expression of the function $\varepsilon$ defined in Eq. (6) is not known. For this reason we limit ourselves to the infinitesimal approximation of the octahedral shear strain i.e.

$\varepsilon=\left(\operatorname{tr}\left(E^{2}\right)-\frac{1}{3}(\operatorname{tr} E)^{2}\right)^{1 / 2}$

The choice of this function has no influence on the general theoretical framework presented here for the tissue differentiation. Evaluation of different mathematical expressions of the stimulus is outside the scope of this study. Nevertheless, the stimulus chosen here and defined in Eq. (24) must correspond to (6) for infinitesimal strains, which is the case as long as the mechanical properties of the tissue correspond to bone and the strain remains moderate.

\section{Identification of the model parameters}

The first part of the identification is the determination of the strain-energy functions for the bone and the fibrous tissue. The strain-energy function chosen has the same form for the two materials and is given by Curnier (1994):

$W_{f, b}=\lambda_{f, b}(J-1-\ln (J))+\mu_{f, b} t r E^{2}$

With (25) and Eq. (2) the strain-energy function of the composite becomes:

$W=\left[\kappa \lambda_{f}+(1-\kappa) \lambda_{b}\right][J-1-\ln (J)]+\left[\kappa \mu_{f}+(1-\kappa) \mu_{b}\right] t r E^{2}$

where $\lambda$ and $\mu$ are the Lamé constants and $J=\operatorname{det} F$ is the determinant of the deformation gradient $F$. There is a one-to-one relationship between the two Lamé constants, and the Young's modulus and the Poisson's ratio. This strain-energy function is admissible in the sense of the definition given previously as long as $\lambda>\mu / 12$. The advantage of the present law is its stable behavior for large 


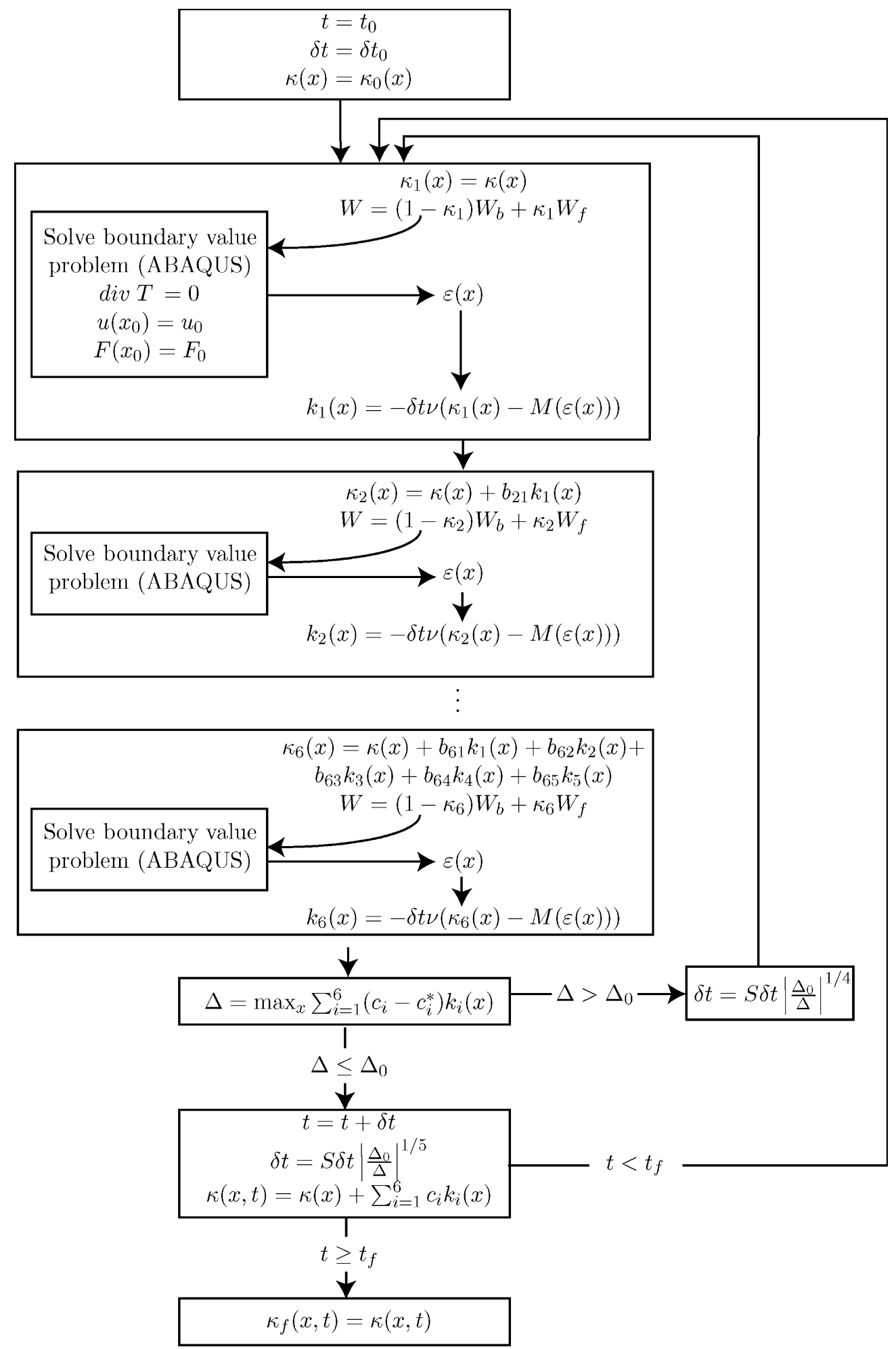

Fig. 3. Schematic description of the numerical algorithm used. The resolution of the initial-value problem was based on a modified Runge-Kutta method. This method required six resolutions of the initial-value problem for each time step $\delta t$ with the problem geometry and boundary conditions. The coefficients $b_{i j}, c_{i}$ and $c_{i}^{\star}$ may be found in Press et al. (1993). The parameter $S$ is a safety factor limiting the risks of failure of the integration for a given step. In this study $S$ was set to 0.9 , the value of the precision criterion $\Delta_{0}$ was set to $10^{-2}$ and the final time $t_{f}$ to 2 months

compression strains. In this model, a good behavior of the bone during compression is needed because the composite may undergo significant strains when the bone-tissue fraction is small. The material Young's modulus and the Poisson's ratio of bone are well defined (Hayes 1991; Reilly et al. 
1974; Rice et al. 1988). For the fibrous tissue, $\lambda_{f}$ and $\mu_{f}$ are obtained by fitting the stress-strain law derived from relation (25) to experimental data (Hori and Lewis 1982).

The model also includes three parameters for the law of evolution (7), the evolution rate $v$ and the two thresholds $\varepsilon_{\min }$ and $\varepsilon_{\max }$. In order to determine these parameters, an axisymmetric finite element (FE) model was developed with the same geometry and loading conditions as the experiment of Jasty et al. (1997). The three parameters are varied until we obtained the same thickness of fibrous tissue as Jasty et al. for micromotions of $20 \mu \mathrm{m}$ and $150 \mu \mathrm{m}$ after 3 weeks of evolution. The identification gives: $\varepsilon_{\text {min }}=2.310^{-3}, \varepsilon_{\text {max }}=8.210^{-3}$ and $v=5$ weeks $^{-1}$.

\section{Application to an idealized model of the hip implant system}

The hip is modeled as a hollow cylinder with a metal prosthesis in its central part. The bone geometry was axisymmetric but the model was reconstructed three-dimensionally. The model includes 1800 eight-node hexahedral elements and account for about 8000 degrees of freedom. The bone was divided into two regions, the cortical bone outside and the cancellous bone inside (in contact with the prosthesis). To precisely determine the bone-fibrous tissue interface, the mesh was refined in the region around the implant. The loading conditions were asymmetric and corresponded to a momentum on the superior part of the prosthesis with the base of the external cylinder fixed (Fig. 4). The mechanical properties of the cancellous bone were dependent on the relative density $\phi$. Three different initial densities were investigated: $\phi=0.14,0.12$, and 0.10 . A density $\phi=0.14$ corresponds to a healthy cancellous bone $(E=294 \mathrm{MPa})$ while a density of $\phi=0.10$ corresponds to half of the mechanical properties of the healthy bone $(E=150 \mathrm{MPa})$. The prosthesis was assumed to be in contact with the cancellous bone. The coefficient of friction was set to $\mu=0.6$.

Numerical results are shown in Fig. 5. The state of the evolution after 8 weeks strongly depended on the initial bone density. For a healthy initial bone density, only a limited propagation of the fibrous tissue was observed in the proximal part of the bone. For lower initial density, the extent of the fibrous tissue around the implant increased and a thick fibrous membrane was present around the implant. The thickness of the fibrous tissue was not constant all around the implant. There was no fibrous tissue in the upper left and lower right part of the interface. The mean thickness of the fibrous tissue was about $1.5 \mathrm{~mm}$ (Fig. 5). Results showed also that the fibrous formation has a wave-like propagation mode (Fig. 5). This is also apparent on Fig. 6 with the temporal evolution of some nodes chosen in the cancellous bone around the implant. Some regions of the bone became fibrous during the week following the loading. For other regions, the fibrous formation started at a later stage of the evolution while for some regions the tissue was fibrous for a limited time period during the evolution. When the model was unloaded, the tissue returned to bone at the same speed for all the regions.

\section{Discussion}

A model of tissue differentiation at the bone-implant interface was proposed. The conservation equations showed that the deformation of the solid composite may be calculated using the classical methods used in continuum mechanics. This important result is due to the very different characteristic time involved in the biological process. The characteristic time of the deformation is the minute whereas the characteristic time of the tissue remodeling is the week.

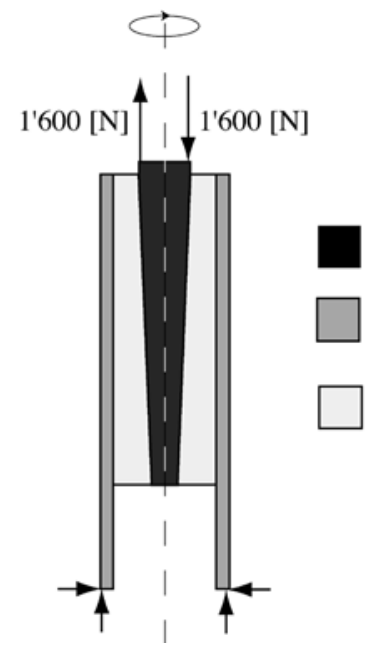

Prosthesis

$\mathrm{E}=200 \mathrm{GPa}, v=0.3$

Cortical bone

$\mathrm{E}=15 \mathrm{GPa}, v=0.3$

Cancellous bone

$\mathrm{E}=\mathrm{E}_{0} \phi^{2}, v=0.3$
Fig. 4. Axisymmetric model of the hip with the boundary conditions. The model was reconstructed three-dimensionally, and mesh was generated with 8-node hexahedral elements. Mechanical properties used for the prosthesis and the bone are given in the figure, where $\mathrm{E}$ is the Young modulus and $v$ the Poisson's ratio. Mechanical properties of the cancellous bone depended on the square of the relative density of the bone $\phi$ with $\mathrm{E}_{0}=15 \mathrm{GPa}(\mathrm{E}=$ $294 \mathrm{MPa}$ for $\phi=0.14$ and $\mathrm{E}=150 \mathrm{MPa}$ for $\phi=0.10$ ) 
A

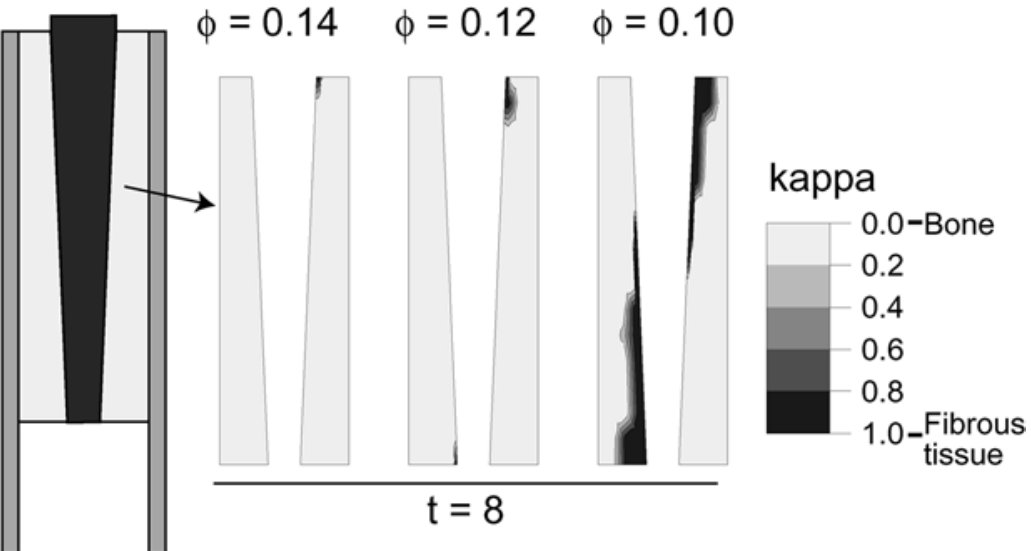

B

$\phi=0.10$

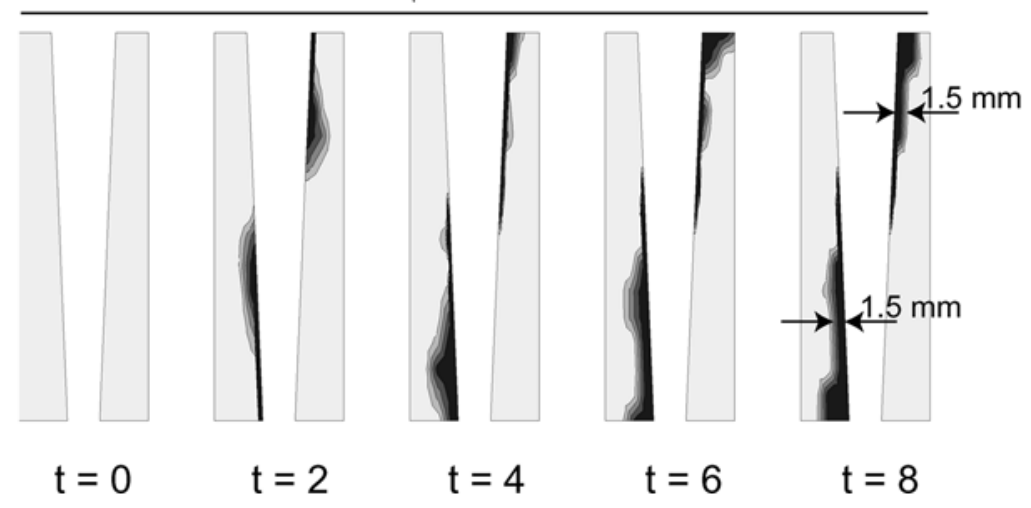

Fig. 5a, b. Evolution of the fibrous tissue at the bone-implant interface. a Results of the evolution after 8 weeks for different initial cancellous bone density; $\mathbf{b}$ temporal evolution of the interface for $\phi=0.14$

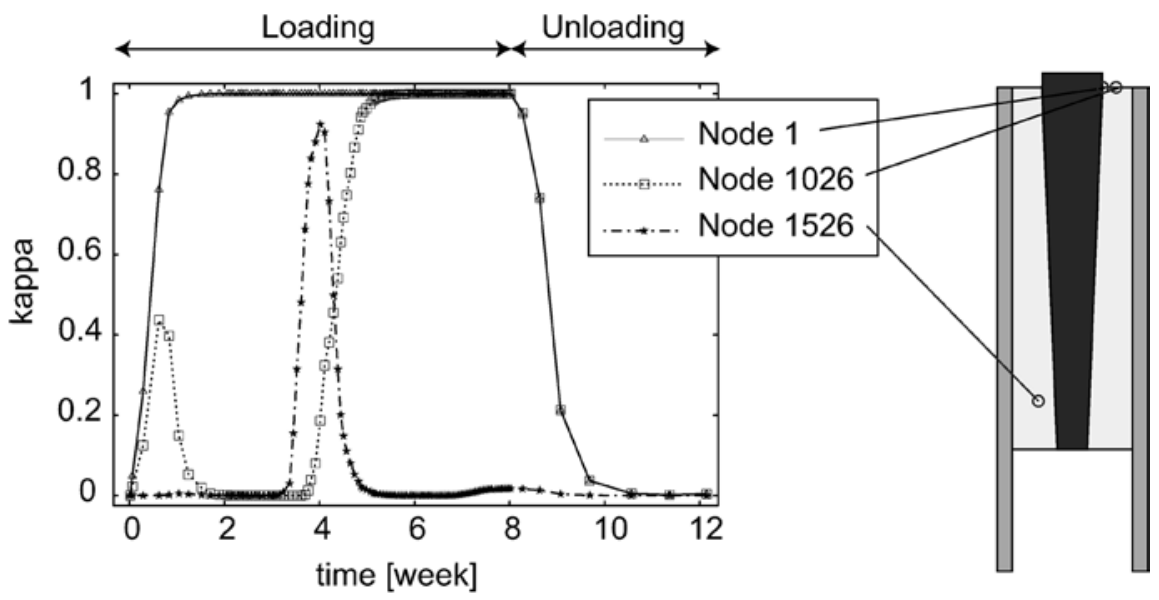

Fig. 6. Temporal evolution of different nodes in the cancellous bone around the prosthesis in the case $\phi=0.10$. From $t=0$ to $t=8$ weeks the load was applied on the upper part of the prosthesis and then the load was removed

The application of the remodeling algorithm to the model of the hip gives results in agreement with clinical observations. The model geometry was simplified but the size of the bone and of the implant was chosen to be realistic as well as the boundary conditions. The different initial density of the cancellous bone was chosen to reflect different initial bone quality or patient bone stock. A relative density of $\phi=0.14$ corresponds to healthy cancellous bone present in young persons and a relative density of $\phi=0.10$ corresponds to poor bone quality for example due to osteoporosis (Jones et al. 1987). Results showed that healthy initial bone quality provided a good initial stability. In this case, only little fibrous formation was observed, which is in agreement with experimental observations 
(Brunski et al. 1979; Levy et al. 1996). For poor initial bone quality $(\phi=0.10)$, the thickness of the fibrous tissue predicted with the present model as well as the time needed to produce this fibrous tissue are similar to the values reported by Cook et al. (1988) for prosthesis retrieved from old patients.

The application of the model proposed here has some interesting results related to the temporal evolution of the fibrous interface. The first observation is that the fibrous tissue was only observed in the bone in contact with the prosthesis. This is an important point since in the model all the cancellous bone around the implant has the potential to be transformed into a fibrous tissue. The second observation is that the propagation of the fibrous interface is similar to the propagation of a cracks along the bone-implant interface. This can be explained by the important difference of the mechanical properties of the fibrous tissue and of the cancellous bone. When a region becomes fibrous, the adjacent bone section supports the load. This provokes a significant strain in this bone section, which in turn becomes fibrous.

For the long-term evolution, there is a fibrous membrane on almost half of the prosthesis in the case of a low initial bone density. Bone is present in the upper left and lower right regions. This is not in complete agreement with the clinical observations of a fibrous encapsulation around all of the prosthesis. Two reasons may explain this discrepancy. First, the contact law does not take into account tensile forces. This implies that when there is no more contact, no strain is generated within the bone matrix. It seems reasonable to assume that the bone-implant interface supports some tensile forces. The second reason is that only one loading condition is represented here. In real situations, the implant is submitted to many different loading conditions representing different daily activities.

The formulation of the mechanical stimuli is of central interest for any remodeling models. The present stimulus was chosen to reflect what the cells feel. Recent work on bone cells in vitro (Pioletti et al. 2003) shows that mechanically stimulated osteoblasts have completely different gene expression than non-stimulated osteoblasts, with bone mineralizing factors being down-regulated. This indicates that the deformation of the cells may lead to non-mineralized bone and then to the formation of a fibrous tissue. Another important point is that the cells in contact with the prosthesis at the bone surface are not able to support all the deformation. A cell with a typical size of $10 \mu \mathrm{m}$ is not able to support the shear strain induced by a micromotion of $150 \mu \mathrm{m}$. The strain would be too high damaging the cell. These two points confirm the hypothesis chosen here that the stimulus of differentiation is the deformation of the matrix around the implant, which in turn induces the deformation of the cells present into this matrix. Other mathematical expressions of the stimulus may be used in the same theoretical framework. For example, a formulation of the stimulus corresponding to the octahedric shear strain in finite deformation would be an improvement of the model.

This model may be useful for a better understanding of the processes of failure of orthopedic implants. A typical application of the model will be for designing new implants that minimize the formation of fibrous tissue.

\section{References}

Aspenberg, P.; Herbertsson, P.: Periprosthetic bone resorption: particles versus movement. J Bone Joint Surg Br 78 (1996) 641-466

Ball, J.: Convexity conditions and existence theorems in nonlinear elasticity. Arch Ration Anal 63 (1977) 337-403

Brunski, J.B.; Moccia, A.F.J.; Pollack, S.R.; Korostoff, E.; Trachtenberg, D.I.: The influence of functional use of endosseous dental implants on the tissue-implant interface. I. Histological aspects. J Dent Res 58 (1979) 19531969

Carter, D.R.; Beaupre, G.S.; Giori, N.J.; Helms, J.A.: Mechanobiology of skeletal regeneration. Clin Orthop, 355 Suppl (1998) S41-S55

Carter, D.R.; Blenman, P.R.; Beaupre, G.S.: Correlations between mechanical stress history and tissue differentiation in initial fracture healing. J Orthop Res 6 (1988) 736-748

Cook, S.D.; Thomas, K.A.; Haddad, R.J.J.: Histologic analysis of retrieved human porous-coated total joint components. Clin Orthop 234 (1988) 90-101

Cowin, S.; Hegedus, D.: Bone remodeling, I: A theory of adaptative elasticity. J Elasticity 6 (1976) 313-326

Curnier, A.: Computational methods in solid mechanics. Solid mechanics and its applications, vol 29. Kluwer, Dordrecht (1994)

Farron, A.; Rakotomanana, R.L.; Zambelli, P.Y.; Leyvraz, P.F.: Total knee prosthesis. Clinical and numerical study of micromovements of the tibial implant. Rev Chir Orthop Reparatrice Appar Mot 80 (1995) 28-35

Flory, P.: Thermodynamic relations for high elastic materials. Trans Faraday Soc 57 (1961) 829-838

Giori, N.J.; Ryd, L.; Carter, D.R.: Mechanical influences on tissue differentiation at bone-cement interfaces. J Arthroplasty 10 (1995) 514-522

Goodman, S.B.: The effects of micromotion and particulate materials on tissue differentiation. Bone chamber studies in rabbits. Acta Orthop Scand Suppl, 258 (1994) 1-43 
Hayes, W.C.: Biomechanics of cortical and trabecular bone: implications for assessment of fracture risk. In: Mow VC, Hayes WC (Eds) Basic orthopaedic biomechanics. Raven Press, New York, (1991) pp. 93-142

Holzapfel, G.: Non-linear solid mechanics: a continuum approach for engineering. Wiley, Chichester (2000)

Hori, R.Y.; Lewis, J.L.: Mechanical properties of the fibrous tissue found at the bone-cement interface following total joint replacement. J Biomed Mater Res 16 (1982) 911-927

Jasty, M.; Bragdon, C.; Burke, D.; O'Connor, D.; Lowenstein, J.; Harris, W.H.: In vivo skeletal responses to poroussurfaced implants subjected to small induced motions. J Bone Joint Surg Am 79 (1997) 707-714

Jones, C.D.; Laval-Jeantet, A.M.; Laval-Jeantet, M.H.; Genant H.K.: Importance of measurement of spongious vertebral bone mineral density in the assessment of osteoporosis. Bone 8 (1987) 201-206

Levy, D.; Deporter, D.A.; Pilliar, R.M.; Watson, P.A.; Valiquette, N.: Initial healing in the dog of submerged versus non-submerged porous-coated endosseous dental implants. Clin Oral Implants Res 7 (1996) 101-110

Pioletti, D.P.; Müller, J.; Rakotomanana, L.; Corbeil, J.; Wild, E.: Effect of micro-mechanical stimulations on osteoblasts: development of a device simulating the mechanical situation at the bone-implant interface. J Biomech 36 (2003) 131-135

Pioletti, D.P.; Takei, H.; Kwon, S.Y.; Wood, D.; Sung, K.L.: The cytotoxic effect of titanium particles phagocytosed by osteoblasts. J Biomed Mater Res 46 (1999) 399-407

Prendergast, P.J.; Huiskes, R.; Soballe, K.: ESB research award 1996. Biophysical stimuli on cells during tissue differentiation at implant interfaces. J Biomech 30 (1997) 539-548

Press, W.; Teukolsky, S.; Vetterling, W.; Flannery B.: Numerical recipes in C: the art of scientific computing, 2nd edn. Cambridge University Press, Cambridge (1993)

Reilly, D.T.; Burstein, A.H.; Frankel, V.H.: The elastic modulus for bone. J Biomech 7 (1974) 271-275

Rice, J.C.; Cowin, S.C.; Bowman, J.A.: On the dependence of the elasticity and strength of cancellous bone on apparent density. J Biomech 21 (1988) 155-168

Søballe, K.; Brockstedt-Rasmussen, H.; Hansen, E.S.; Bunger, C.: Hydroxyapatite coating modifies implant membrane formation. Controlled micromotion studied in dogs. Acta Orthop Scand 63 (1992a) 128-140

Søballe, K.; Hansen, E.S.; Brockstedt-Rasmussen, H.; Jorgensen, P.H.; Bunger, C.: Tissue ingrowth into titanium and hydroxyapatite-coated implants during stable and unstable mechanical conditions. J Orthop Res 10 (1992b) 285-299

Skripitz, R.; Aspenberg, P.: Pressure-induced periprosthetic osteolysis: a rat model. J Orthop Res 18 (2000) 481-484

Terrier, A.; Rakotomanana, R.L.; Ramaniraka, A.N.; Leyvraz, P.F.: Adaptation models of anisotropic bone. Comput Methods Biomech Biomed Eng 1 (1997) 47-59

van der Vis, H.M.; Aspenberg, P.; de Kleine, R.; Tigchelaar, W.; van Noorden, C.J.: Short periods of oscillating fluid pressure directed at a titanium-bone interface in rabbits lead to bone lysis. Acta Orthop Scand 69 (1998a) 5-10

van der Vis, H.M.; Aspenberg, P.; Marti, R.K.; Tigchelaar, W.; van Noorden, C.J.: Fluid pressure causes bone resorption in a rabbit model of prosthetic loosening. Clin Orthop 350 (1998b) 201-208

van der Vis, H.M.; Aspenberg, P.; Tigchelaar, W.; van Noorden, C.J.: Mechanical compression of a fibrous membrane surrounding bone causes bone resorption. Acta Histochem 101 (1999) 203-212

Weinans, H.; Huiskes, R.; Grootenboer, H.J.: Trends of mechanical consequences and modeling of a fibrous membrane around femoral hip prostheses. J Biomech 23 (1990) 991-1000

Weinans, H.; Huiskes, R.; Grootenboer, H.J.: Effects of fit and bonding characteristics of femoral stems on adaptive bone remodeling. J Biomech Eng 116 (1994) 393-400

Yuan, X.; Ryd, L.; Huiskes, R.: Wear particle diffusion and tissue differentiation in tka implant fibrous interfaces. J Biomech 33 (2000) 1279-1286 